\title{
„Mein Coach für Wohlbefinden und Gesundheit“. User Experience von E-Health-Technologien
}

\author{
Jasmin Niess, Sarah Diefenbach \\ Department Psychologie, Ludwig-Maximilians-Universität München
}

\section{Zusammenfassung}

E-Health-Technologien können das individuelle Gesundheitsverhalten unterstützen und fördern. Doch es bedarf einer Integration von psychologischem und technischem Wissen, um dieses Potential auszuschöpfen. Ein wichtiger Aspekt ist hierbei der kurative Dialog zwischen Produkt/ Coach und Nutzer/ Klient. Anhand einer qualitativen Nutzerbefragung untersucht der Beitrag die Wahrnehmung der Interaktion und Kommunikation von und mit E-Health-Technologien und skizziert Aufgaben zukünftiger Forschung.

\section{Einleitung}

E-Health-Technologien (z.B. Smartphone-Apps wie OptimizeMe, Fitness-Gadgets wie Fitbit) bieten neue Potenziale für die Unterstützung von gesundheitsförderlichem Verhalten (Conroy et al., 2013). Das Produkt wird zum Coach, der Möglichkeiten zur Verhaltensoptimierung aufzeigt, in Bereichen wie z.B. Gewichtsreduktion, Stressmanagement, Schlafverhalten. Es fehlen jedoch noch adäquate Modelle für die Konzeption von E-Health-Interventionen, welche psychologisches Wissen zu Veränderungsprozessen und das Potential interaktiver Technologien kombinieren (Free et al., 2013). Auch das Erleben des Nutzers (User Experience, UX) und relevante Faktoren für Motivation und Nutzungserfolg sind noch nicht ausreichend erforscht. Ein wichtiger Aspekt scheint hier der Dialog zwischen Produkt/Coach und Nutzer/Klient. Er gilt in der traditionellen Therapie als wesentlich für den Veränderungserfolg (z.B. Lutz, 2010). Im Fall von interaktiven Produkten könnten relevante Elemente des Dialogs und Kommunikationsstils z.B. die Art von Feedback und Verbesserungshinweisen sein. Ziel unserer Forschung ist es die Wahrnehmung der Interaktion und Kommunikation zwischen Nutzer und Produkt zu untersuchen. Hierdurch wollen wir bestehendes Wissen zur User Experience von E-Health-Technologien erweitern und Hinweise für die Gestaltung ableiten. 


\section{Interviews mit Nutzern von E-Health-Technologien}

In einer ersten explorativen Studie führten wir 14 Interviews mit Nutzern von E-HealthTechnologien (im Sinne eines erweiterten Gesundheits-Begriffs verstehen wir hierunter interaktive Produkte zur Veränderungsunterstützung bezüglich psychischer und physischer Gesundheit). Das Hauptaugenmerk lag auf der Interaktion und der Kommunikation zwischen den Nutzern und ,ihrem“ Produkt. Die Interviews wurden transkribiert und die berichteten Charaktermerkmale und Kommunikationsstile in Anlehnung an Schulz von Thun (1989) analysiert. Hierbei zeigte sich, dass Nutzer den Kommunikationsstil ,ihres“ Produkts (z.B. belehrend, kooperativ-helfend) wahrnehmen und differenziert beschreiben können. Auch zeigen sich Tendenzen für einen Zusammenhang der Kommunikationsart und Nutzungsdauer. Beispielsweise beschrieben Langzeitnutzer (3 Monate bis 2 Jahre) den Kommunikationsstil eher als neutral und rational (,Zurückhaltend, relativ neutral/ ich hätte sie nicht so lange genutzt, wenn sie mir quasi nicht, im übertragenen Sinn, sympathisch gewesen wäre."). Als wenig motivierend wurde ein belehrender Kommunikationsstil empfunden ( „...ich fände es ziemlich nervig, wenn mir eine App sagen würde: „Jetzt hast du schon wieder so viel gegessen“; oder „du bist über deinem Kalorienlevel“. Ich weiß nicht, das fände ich ein bisschen komisch, wenn der so vorwurfsvoll kommen würde.").

\section{Diskussion und Ausblick}

Basierend auf diesen Einsichten wollen wir in zukünftigen Studien tiefgehendes Wissen zur Relevanz von Interaktion und Kommunikation zwischen Menschen und Selbstverbesserungstools generieren und hierdurch auch konkrete Empfehlungen für die Gestaltung von E-Health-Technologien zur optimalen Unterstützung der User Experience schaffen.

\section{Literaturverzeichnis}

Conroy, D. E., Yang, C. H., \& Maher, J. P. (2014). Behavior change techniques in top-ranked mobile apps for physical activity. American journal of preventive medicine, 46(6), 649-652.

Free, C., Phillips, G., Galli, L., Watson, L., Felix, L., Edwards, P. \& Haines, A. (2013). The effectiveness of mobile-health technology-based health behaviour change or disease management interventions for health care consumers: a systematic review. PLoSmedicine, 10(1), e1001362

Schulz von Thun, F. (1989). Miteinander reden 2: Stile, Werte und PersönlichkeitsentwicklungDifferentielle Psychologie der Kommunikation. Reinbek: Rowohlt.

Lutz, W. (Ed.). (2010). Lehrbuch Psychotherapie. Huber.

\section{Kontaktinformationen}

Jasmin Niess, Sarah Diefenbach

Ludwig-Maximilians-Universität München, Department Psychologie

Leopoldstraße 13, 80802 München, Deutschland

\{jasmin.niess; sarah.diefenbach\}@psy.lmu.de 